\title{
Subretinal alteplase injections in massive subretinal hemorrhage due to age-related macular degeneration: A case report series
}

\author{
RADU OCHINCIUC $^{1 *}$, FLORIAN BALTA ${ }^{2}$, DANIEL CONSTANTIN BRANISTEANU ${ }^{3}$, \\ MARIAN BURCEA $^{2 *}$, MIHAIL ZEMBA ${ }^{2 *}$, ULIANA OCHINCIUC ${ }^{4}$ and RAMONA BARAC ${ }^{2}$ \\ ${ }^{1}$ Department of Ophthalmology, 'Victor Babes' University of Medicine and Pharmacy, 300041 Timisoara; \\ 2 Department of Ophthalmology, 'Carol Davila' University of Medicine and Pharmacy, 050474 Bucharest; \\ ${ }^{3}$ Department of Ophthalmology, 'Grigore T. Popa' University of Medicine and Pharmacy, 700115 Iasi; \\ ${ }^{4}$ Department of Ophthalmology, 'Dr. Carol Davila' Central Military Emergency \\ University Hospital, 010825 Bucharest, Romania
}

Received August 26, 2020; Accepted September 24, 2020

DOI: $10.3892 /$ etm.2020.9338

\begin{abstract}
This report describes a series of cases with massive subretinal hemorrhage (SRH) due to age-related macular degeneration (AMD) treated by subretinal alteplase injections. In all cases, the surgical technique consisted in 25-gauge pars plana vitrectomy (PPV) and alteplase injection under the retina using a 38-gauge cannula. After the fluid-gas exchange, bevacizumab injection was performed in all patients. Three cases of SRH in which this technique was used, as well as their evolution at one week and one month postoperatively are described. Visual acuity was hand motion in all three cases at presentation. After surgery, a significant anatomical and functional improvement was noted in all cases. One month postoperatively, none of the patients had blood under the macula, and visual acuities significantly improved to 0.8 , 0.2 and 0.16 (decimal fraction). A consistent reduction of central retinal thickness was observed on optical coherence
\end{abstract}

Correspondence to: Dr Florian Balta, Department of Ophthalmology, 'Carol Davila' University of Medicine and Pharmacy, 37 Dionisie Lupu Street, 050474 Bucharest, Romania

E-mail: florian.balta@umfcd.ro

Dr Daniel Constantin Branisteanu, Department of Ophthalmology, 'Grigore T. Popa' University of Medicine and Pharmacy, 16 University Street, 700115 Iasi, Romania

E-mail: daniel.branisteanu@umfiasi.ro

*Contributed equally

Abbreviations: AMD, age-related macular degeneration; BCVA, best-corrected visual acuity; DNA, deoxyribonucleic acid; OCT, optical coherence tomography; PPV, pars plana vitrectomy; RPE, retinal pigment epithelium; rTPA, recombinant tissue plasminogen activator; SD-OCT, spectral domain optical coherence tomography; SRH, subretinal hemorrhage; VEGF, vascular endothelial growth factor

Key words: subretinal injection, subretinal hemorrhage, alteplase, age-related macular degeneration tomography (OCT) from the first week postoperatively. No intra and postoperative complications were noted. Subretinal alteplase injection proved as a viable solution in these severe SRH with early presentation. There was no need to change the systemic anticoagulant and antiaggregant therapy. Bevacizumab intravitreal injection at the end of surgery has an important role in preventing further bleeding.

\section{Introduction}

Massive subretinal hemorrhage ( $\mathrm{SRH}$ ) is a devastating complication that can occur in patients with both wet and dry age-related macular degeneration (AMD). Due to the toxic effect of the accumulated iron compounds on the photoreceptors and retinal pigment epithelium (RPE), the therapeutic solution must be found very quickly, otherwise, the visual prognosis is reserved (1-3). Subretinal injections with recombinant tissue plasminogen activator (rTPA) have been used for some time and the results are highly variable from case to case.

Alteplase is a thrombolytic agent, a glycoprotein, produced by recombinant deoxyribonucleic acid (DNA) synthesis in cell culture (4). It is approved by Food and Drug Administration for intravenous administration in acute ischemic stroke, pulmonary embolism, acute myocardial infarction, and occluded catheters $(5,6)$. From its inactive form, it becomes active after fibrin coupling, which eventually results in the transformation of plasminogen into plasmin.

Without treatment, in a massive SRH, in the most common cases, the final visual acuity of the patients is only light perception. The subretinal use of rTPA led to the improvement of these results $(7,8)$, despite possible retinal toxicity observed in cats and rabbits $(9,10)$. Moreover, using immunostaining with antibodies against brain-specific home box/POU domain protein 3a (Brn3a), in a mouse model of glaucoma, a degeneration of retinal ganglion cells was observed, determined by up-regulating of the tissue plasminogen activation (11). Brn3a is a transcription factor expressed in the central and peripheral sensory nervous system such as trigeminal ganglion or retinal ganglion cells (RGCs) in rodents and probably in humans. In the report described herein, it was shown that the increase 
in IOP caused the increase of the proteolytically active tPA, which led to a reduction of Brn3a and RGCs in mice.

However, there is no consensus regarding the indications for this procedure, the dose of the substance, not even the surgical technique. We describe three cases of massive SRH determined by AMD, from our experience, in which we used the subretinal injection of alteplase.

\section{Case reports}

The study was performed accordingly to the Institutional Guidelines of the Ponderas Academic Hospital, Bucharest, Romania. Approval was obtained from the Institutional Review Board and the Ethics Committee of the Ponderas Academic Hospital. All procedures conformed to the tenets of the World Medical Association Declaration of Helsinki. All patients gave their written informed consent.

A series of three cases of SRH are described. In all cases, a 25-gauge pars plana vitrectomy (PPV) with hyaloid removal was performed. In eyes with vitreous hemorrhage, all blood and membranes were removed from the retinal surface. For the subretinal injection, a $1 \mathrm{ml}$ insulin syringe and a 38-gauge cannula were used. Actilyse ${ }^{\circledR} 50 \mathrm{mg}$ (Boehringer Ingelheim International GMBH, Germany) was used. After the powder and solvent were properly mixed according to the instructions, 0.6-0.7 $\mathrm{ml}$ of solution was drawn into the syringe. On average $0.3 \mathrm{ml}$ of the substance was injected through the lower part of the macula, until a bullous retinal detachment was obtained. Depending on the size of the clot, more or less of the substance was injected or a second injection was made in another part of the macula. In some cases, a quick dislocation of the clot through the injected site was observed. After total fluid-air exchange, $1.25 \mathrm{mg} / 0.05 \mathrm{ml}$ bevacizumab (Avastin ${ }^{\circledR}$, Roche, Switzerland) injection was intravitreally injected in all patients (12). Face-down position was recommended in all cases for the next $24 \mathrm{~h}$. Patients were evaluated on the first day, 1 week and 1 month after surgery.

Case 1. A 74-year old male with neovascular AMD in both eyes, with anti-vascular endothelial growth factor (VEGF) therapy in the right one, presented due to a sudden decrease of visual acuity and central scotoma for 2-3 days in his right eye. Due to cardiovascular risk, the patient was treated with anti-aggregating agents and oral anticoagulants for several years. Best-corrected visual acuity (BCVA) at the presentation was hand motion. At the fundoscopic examination, a massive SRH was observed affecting the entire macular surface. Optical coherence tomography (OCT) revealed a significant retinal thickening. The patient was submitted to PPV, with subretinal rTPA injection and intravitreal bevacizumab. Fluid-gas exchange was performed at the end of the surgery. Due to gas and diffuse vitreous hemorrhage, weakly transparent, BCVA was hand motion the first 2 weeks postoperatively. One month after the surgery BCVA was 0.8 (decimal fraction), with a significant improvement of the fundoscopic and spectral domain optical coherence tomography (SD-OCT) examination (Fig. 1). Clinical examination revealed the presence of the blood clot inferior, below the peripheral retina. The patient followed regular checkups, with intravitreal injections of bevacizumab as needed.
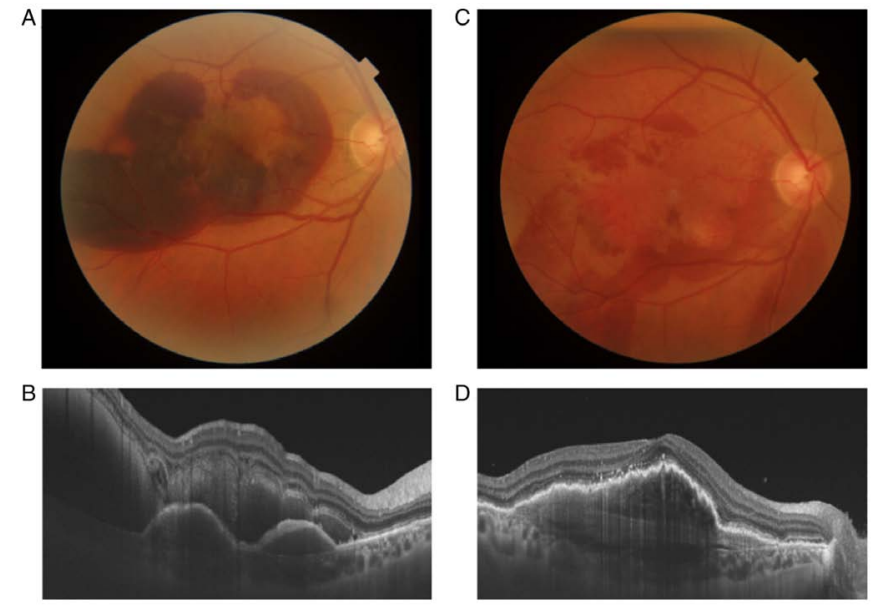

Figure 1. Clinical and optical coherence tomography appearance of Case 1. (A and B) Fundus and optical coherence tomography (OCT) of the macula at the presentation, showing a massive subretinal hemorrhage and a significant retinal thickening, intraretinal fluid, moderate reflective material under neuroepithelium and retinal pigment epithelial detachment (PED). (C) Fundus of the same case, one month after surgery, which shows an almost complete resorption of the hemorrhage from the macula level and the persistence of the air in the upper part. (D) OCT of the macula, one month after surgery, which shows a significant decrease in retinal thickness, lack of subretinal blood and the presence of a PED.

Case 2. A 60-year old male patient was referred with acute vision loss for 2 days in the right eye. Without ophthalmological history, being in treatment with oral anticoagulants for 3 years. BCVA at presentation was hand motion. The fundoscopic examination revealed an important $\mathrm{SRH}$ in the macular area, with a marked increase in retinal thickness on SD-OCT. PPV and subretinal rTPA injection were performed on the same day. Intraoperatively, a slight movement of the clot was already observed. Seven days after the surgery the BCVA was 0.05. The fundoscopic aspect improved significantly, a few small areas of blood below the retina and under the RPE persisted. On SD-OCT the retinal thickness is close to normal, foveolar depression is also observed. In one month no postoperative complications appeared, BCVA was 0.2 , fundoscopic and SD-OCT appearance were preserved or even small improvements were observed (Fig. 2).

Case 3. A 70-year old woman with neovascular AMD, treated with anti-VEGF agents, was referred to our clinic for a severe SRH. The last injection with bevacizumab was 10 months before this event. Visual acuity decreased to hand motion for about 4 days. The clinical aspect of the retina was similar to the previous cases, a massive submacular hemorrhage. On SD-OCT, a subretinal and under RPE hemorrhages were observed. The same surgical steps were followed in this case. BCVA was counting fingers after the first week and 0.16 after one month, the patient was very happy that he returned to visual acuity as before the event. At the fundoscopic examination, the remains of hemorrhage were visualized the first week postoperatively. The SRH disappeared almost completely over a month, an area of RPE atrophy and pigmentary changes remained at the macula level. The SD-OCT showed an RPE detachment, with possible RPE rolls and a significant distortion of the outer layers of the retina after 1 week. The aspect 

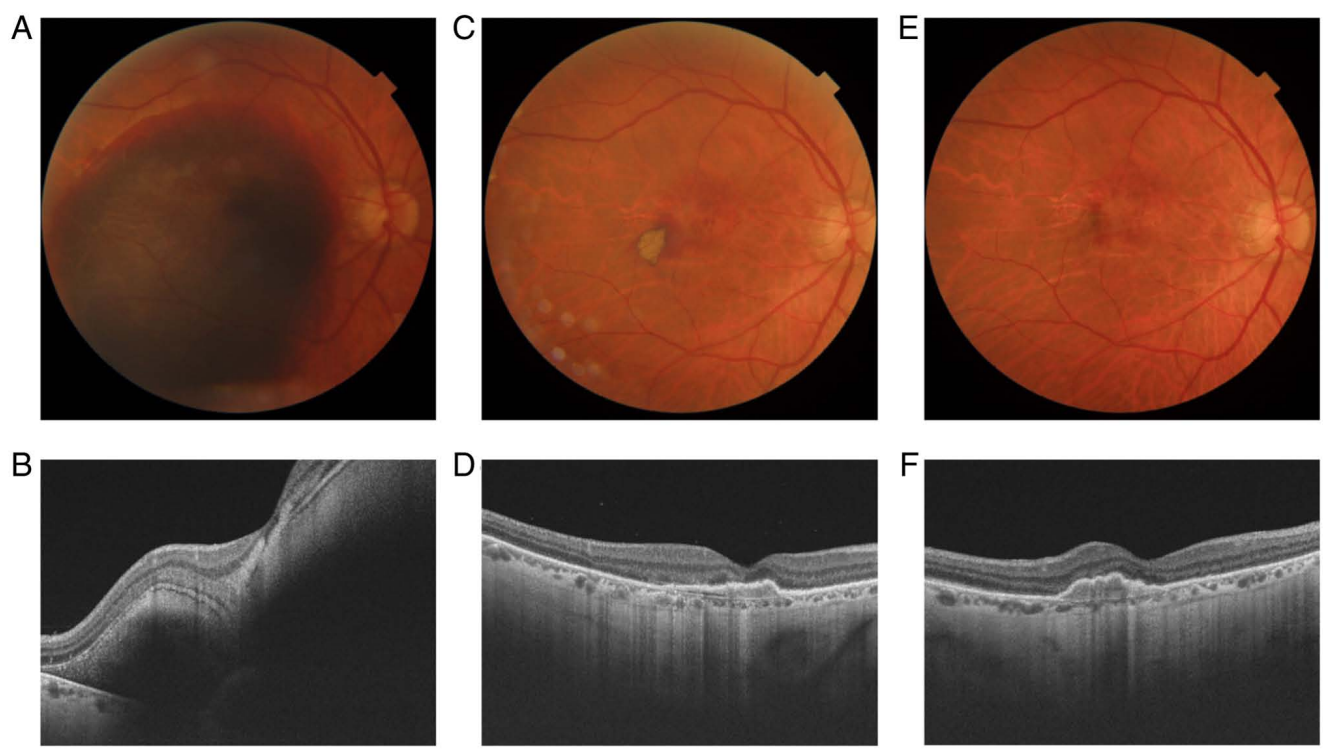

Figure 2. Clinical and optical coherence tomography appearance of Case 2. (A and B) Fundus and optical coherence tomography (OCT) at presentation, showing a massive subretinal hemorrhage, with a significant increase of the retinal thickness. (C and D) One week after the surgery, the fundus shows a significant improvement in clinical appearance, with the persistence of a small amount of dehemoglobinized blood, and a significant reduction of retinal thickness on OCT. (E and F) One month after surgery, fundus reveals some traces of subretinal haemorrhage, and the persistence of retinal pigment epithelial alterations on OCT.
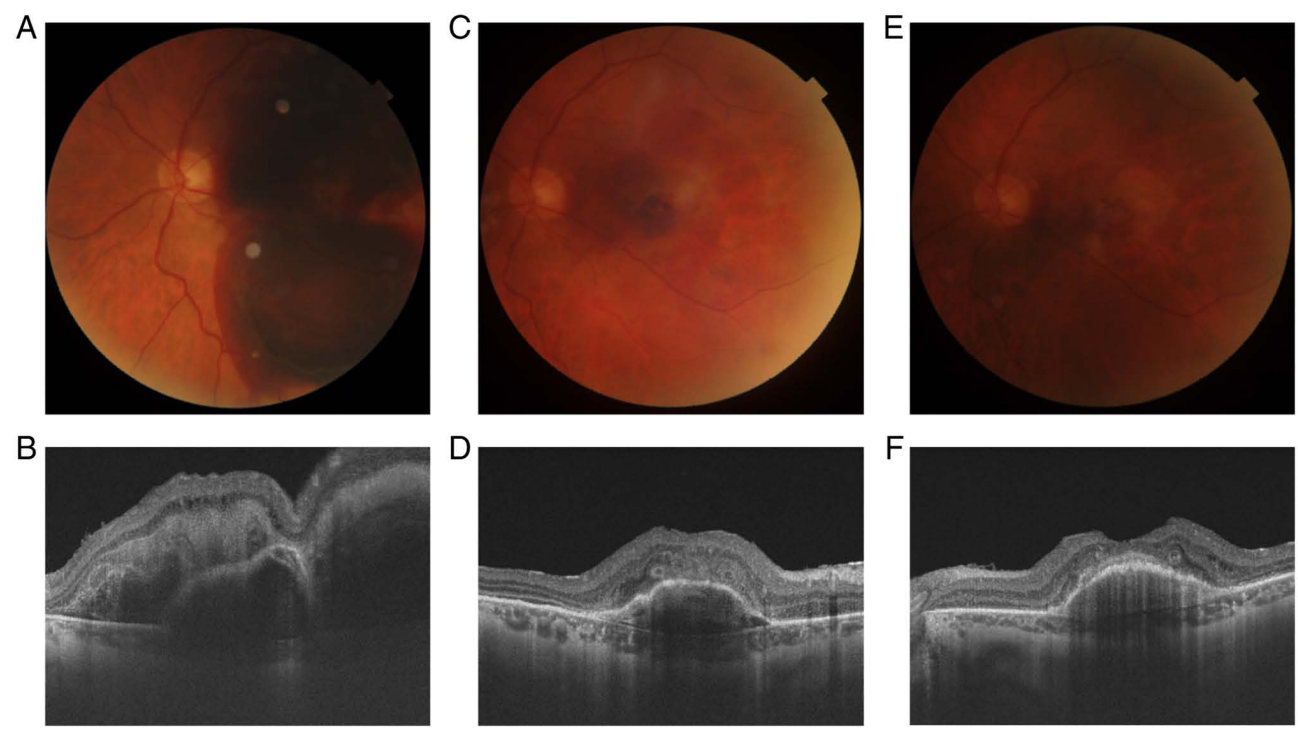

Figure 3. Clinical and optical coherence tomography appearance of Case 3. (A and B) Fundus and optical coherence tomography (OCT) at presentation, showing a massive subretinal hemorrhage, with a significant increase of the retinal thickness, a subretinal hyperreflective tissue mass and intraretinal cystic spaces. (C and D) One week after surgery, persistence of subretinal blood in the center of the macula is observed on the fundus, but with a significant decrease in retinal thickness, with typical age-related macular degeneration changes in the external retinal structures on the OCT. (E and F) One month after surgery, the hemorrhage disappeared almost completely, retinal pigment epithelial (RPE) detachment, with possible RPE rolls on the OCT.

has improved significantly over a month (Fig. 3). The patient followed regular check-ups, with intravitreal injections of bevacizumab or aflibercept as needed.

\section{Discussion}

AMD is one of the most common causes of SRH in adults. Therefore the definitive visual acuity of each patient, regardless of the therapeutic solution chosen, also depends on the degree of macular damage before bleeding. In our practice, we have 9 cases of SRH for which subretinal injections with
rTPA were performed. Eight of them were caused by AMD and a single one by a choroidal neovascular membrane in a high myopic eye. In most cases, the baseline visual acuity was hand motion or light perception and at least counting fingers after the surgery. Case number 1 presented above is the one in which the best postoperative visual acuity was obtained. The postoperative clinical and OCT appearance was spectacular in all cases.

The prognostic factors of postoperative visual acuity was observed to be visual acuity before hemorrhage occurred and the time elapsed since the onset of the SRH. The most frequent 
contraindication for rTPA injection was late presentation. Late presentation means poor visual potential due to neurosensory retinal distress (13). All cases in which a visual acuity greater than counting fingers was obtained, presented in the first 5 days of onset. The maximum duration of the SRH for which we used this technique was 3 weeks, and postoperative visual acuity was counting fingers at $1 \mathrm{~m}$. We did not have any intraoperative complications due to alteplase or other factors. Regarding postoperative complications only one case of rhegmatogenous retinal detachment, caused by a peripheral retinal break, was observed.

Regarding the surgical technique used, we considered that it is one of the most optimal in terms of the applications during the surgery and after it. Probably one of the most widely used methods is for the surgeon to hold the syringe and an assistant to push the plunger. In other words, this technique requires a good collaboration between team members and a good training of the entire team of surgeons and assistant. Another method to inject the alteplase is using the viscous fluid control unit. This is a semiautomated technique that requires an insulin syringe of 1-ml adapted to the viscous fluid control unit and a 41-gauge cannula. This technique probably offers more control and stability than the assisted one. In our opinion what makes our technique more practical and easy to use is that all operative steps can be performed by a single surgeon (14). No additional time is needed for clot lysis or to prepare special injection devices (15). There are no studies that compare the functional results of these surgical techniques, so at the moment the choice is strictly related to the comfort and possibilities of the surgeon. Based on our experience the fluid-air exchange is sufficient for the mobilization of the clot, the injection of an expandable gasis not necessary. The decision to inject an anti-VEGF drug at the end of the operation seems to be quite important, considering the pathophysiological mechanism of the SRH.

In massive hemorrhages, a larger amount of alteplase was needed for clot dislocation and dissolution. However, even in these patients, no dose-dependent toxic effect was observed. Also, no special attitude was taken towards patients treated with anticoagulant or anti-aggregating agents. All the patients followed the treatment recommended by the cardiologist. Moreover, the injection with anti-VEGF agent at the end of the surgery had exactly this purpose, to prevent subsequent bleeding, which did not occur in any of the cases.

Studying the maximum duration of the SRH for which surgery is worthwhile is one of our future goals. Another purpose is a long-term follow-up of patients in whom the subretinal alteplase was injected and their comparison with those in whom a less invasive therapeutic solution was decided.

$\mathrm{SRH}$ is a common complication of the AMD which can lead to irreversible loss of visual acuity. Due to intravitreal injections with anti-VEGF agents, AMD in most patients can be kept under control. Thus, surgery has become an indication only for complicated cases of SRH. Subretinal injection with rTPA is a viable solution in patients with massive SRH who are addressed on time. In our practice, this technique has become the first therapeutic approach that we adopt in such cases.

\section{Acknowledgements}

Not applicable.

\section{Funding}

No funding was received. This research did not receive any specific grant from funding agencies in the public, commercial, or not-for-profit sectors.

\section{Availability of data and materials}

The first author and the corresponding author have full access to all the data and materials in the study, and the data are available from the corresponding author on reasonable request.

\section{Authors' contributions}

$\mathrm{RO}, \mathrm{FB}$ and $\mathrm{RB}$ participated in conceptualization, design and writing of the original draft. $\mathrm{MB}, \mathrm{MZ}$ and $\mathrm{UO}$ were involved in the analysis and visualization of the results, the acquisition of the data and literature research. DSB, RO and FB did a critical interpretation of the data for the study and edited the manuscript. All authors revised the manuscript for important intellectual content, read and approved the final version of the manuscript to be published.

\section{Ethics approval and consent to participate}

The study was retrospectively approved by the Institutional Review Board and the Ethics Committee of Ponderas Academic Hospital (Bucharest, Romania). All procedures conformed to the tenets of the World Medical Association Declaration of Helsinki.

\section{Patient consent for publication}

Written informed consent was obtained from all the patients.

\section{Competing interests}

The authors declare that they have no competing interests.

\section{References}

1. Lee JH, Lee MY and Lee WK: Incidence and risk factors of massive subretinal hemorrhage in retinal angiomatous proliferation. PLoS One 12: e0186272, 2017.

2. Maranduca MA, Branisteanu D, Serban DN, Branisteanu DC, Stoleriu G, Manolache N and Serban IL: Synthesis and physiological implications of melanic pigments. Oncol Lett 17: 4183-4187, 2019.

3. Munteanu M, Rosca C and Stanca H: Sub-inner limiting membrane hemorrhage in a patient with Terson syndrome. Int Ophthalmol 39: 461-464, 2019.

4. Michel P, Lindsay P, Martins S, Pandian JD, Caso V, Kim JS, Bryer A, Anderson C, Feigin V, Sandercock P, et al: Alteplase (recombinant tissue Plasminogen Activator, rt-PA) for the Treatment of Acute Ischemic Stroke. Application for inclusion of a new individual medicine in the WHO Model List of Essential Medicines (EML) For the 2019 WHO Expert Committee on the Selection and Use of Essential Medicines and Canadian Stroke Network. Alteplase for AIS: WHO EML 2019 application. https://www.who.int/selection_medicines/committees/expert/22/ applications/s12.5.2_alteplase.pdf?ua $=1$.

5. Stanca HT, Petrović Z and Munteanu M: Transluminal Nd:YAG laser embolysis - a reasonable method to reperfuse occluded branch retinal arteries. Vojnosanit Pregl 71: 1072-1077, 2014.

6. Harvison PJ: Alteplase. In: xPharm: The Comprehensive Pharmacology Reference. Elsevier Inc., Amsterdam, pp1-5, 2007. 
7. Fine HF, Iranmanesh R, Del Priore LV, Barile GR, Chang LK, Chang S and Schiff WM: Surgical outcomes after massive subretinal hemorrhage secondary to age-related macular degeneration. Retina 30: 1588-1594, 2010.

8. Singh RP, Patel C and Sears JE: Management of subretinal macular haemorrhage by direct administration of tissue plasminogen activator. Br J Ophthalmol 90: 429-431, 2006.

9. Johnson MW, Olsen KR, Hernandez E, Irvine WD and Johnson RN: Retinal toxicity of recombinant tissue plasminogen activator in the rabbit. Arch Ophthalmol 108: 259-263, 1990.

10. Hrach CJ, Johnson MW, Hassan AS, Lei B, Sieving PA and Elner VM: Retinal toxicity of commercial intravitreal tissue plasminogen activator solution in cat eyes. Arch Ophthalmol 118: 659-663, 2000.

11. Chintala SK: Tissue and urokinase plasminogen activators instigate the degeneration of retinal ganglion cells in a mouse model of glaucoma. Exp Eye Res 143: 17-27, 2016.

12. Stanca HT, Stanca S, Tabacaru B, Boruga M and Balta F: Bevacizumab in Wet AMD treatment: A tribute to the thirteen years of experience from the beginning of the anti-VEGF era in Romania. Exp Ther Med 18: 4993-5000, 2019.
13. Moraru AD, Costin D, Moraru RL, Costuleanu M and Brănișteanu DC: Current diagnosis and management strategies in pachychoroid spectrum of diseases (Review). Exp Ther Med 20: 3528-3535, 2020.

14. Haupert CL, McCuen BW, Jaffe GJ, Steuer ER, Cox TA, Toth CA, Fekrat S and Postel EA: Pars plana vitrectomy, subretinal injection of tissue plasminogen activator, and fluid-gas exchange for displacement of thick submacular hemorrhage in age-related macular degeneration. Am J Ophthalmol 131: 208-215, 2001.

15. Novelli FJD, Preti RC, Monteiro MLR, Nóbrega MJ and Takahashi WY: A new method of subretinal injection of tissue plasminogen activator and air in patients with submacular hemorrhage. Retina 37: 1607-1611, 2017.

(i) (8) This work is licensed under a Creative Common Attribution-NonCommercial-NoDerivatives 4.0 International (CC BY-NC-ND 4.0) License. 